\title{
prévision des effondrements de surface à partir des mesures en souterrain
}

\author{
par \\ E. Tincelin \\ École des Mines, Paris \\ R. Deniau \\ P. Sinou \\ D. Leonet \\ D. de Winter \\ Samifer, Briey
}

CHAPITRE I - GENERALITES - POSITION DU PROBLEME

I-1 - Stabilité des stots

I-1-1- Méthode d'exploitation - Les conséquences au jour

Les mines de fer de lorraine exploitent un gisement en plateure, dont la profondeur varie de 50 à 280 mètres, et dont 1a puissance moyenne des couches exploitées est voisine de $4,50 \mathrm{~m}$. La méthode générale utilisée est celle des chambres et piliers avec foudroyage. Nous ne redonnerons pas ici les détails de cette méthođe bien connue. Nous rappellerons simplement qu'elle aboutit à l'enlèvement du minerai en place, avec un taux moyen de $85 \%$, et que les terrains sus-jacents sont, en général, volontairement foudroyés. (figure 1)

Cette opération se trađuit par un affaissement de surface important, étant donné qu'aucun remblayage n'est pratiqué. Dans le paragraphe I-3, nous donnerons quelques précisions sur les lois physiques de ces affaissements, qui ont été mises en évidence à la suite des mesures systématiques réalisées par Samifer. Disons simplement qu'à la stabilisation, le ratio des affaissements par rapport à la hauteur du vide minier comblé, est en général de $50 \%$.

\section{I-1-2 -Problème posế par les agglomérations}

Il est évident que l'exploitation telle que nous venons de la décrire, pose un problème de stabilité pour les constructions du jour sus-jacentes. En règle générale, les agglomérations sont donc protégées, c'est-à-dire qu'on cherche à réaliser une exploitation qui laisse subsister des vides miniers stables, de sorte que les affaissements des terrains soient nuls ou au moins sans effet sur les constructions.

Fig.1 Schéma de principe de l'exploitation par chambres et piliers avec dépilage et foudroyage sur quilles résiduelles

Remarque importante : Ce croquis, ètabli pour montrer la progression de l'exploitation d'une recoupe, ne doit pas être pris comme modèle pour une exploitation reelle. Dans la pratique, il faut éviter à tout prix d'explorter simultanément des recoupes dans des piliers voisıns.
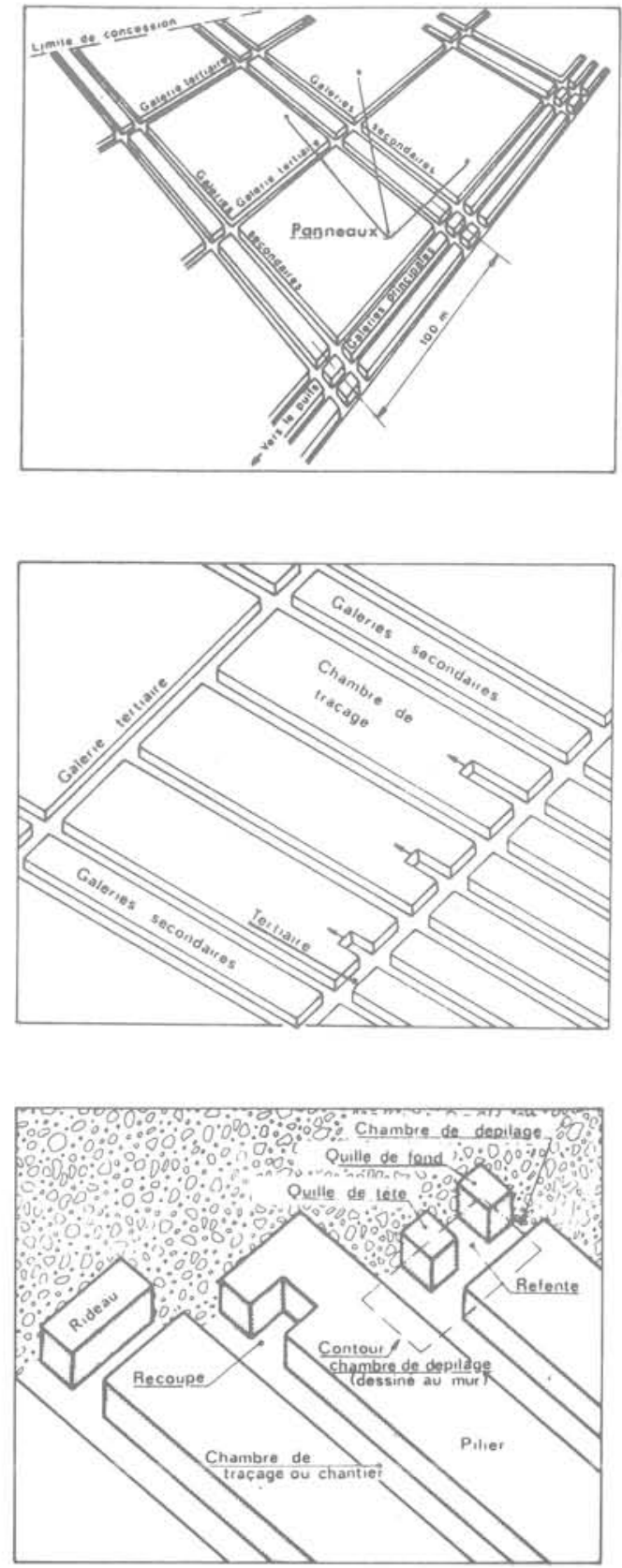
Ainsi, au-dessous de chaque agglomeration, on définit, à partir des plans de surface, la zone qui au fond devra être exploitée avec la méthode particulière.

Cette zone s'appelle, dans le langage des mineurs, le stot, ou stot de protection.

\section{I-1-3 -Règles d'établissement des stots - Mesures d'affaissement de surface}

Le problème des stots de protection s'est posé aux exploitants des mines de fer dès les premiers temps de l'exploitation industrielle du gisement ferrifère lorrain.

Ce problème a donc reçu des solutions qui étaient apportées en fonction des connaissances possédées par les ingénieurs qui dessinaient les plans et dirigaient les travaux.

On a donc, dès le départ formulé des règles empiriques basées sur l'expérience. Les services techniques de la profession ont évidemment cherché à perfectionner ces règles en se livrant à des mesures d'affaissement au-dessus des zones đépilées. Ces mesures étaient effectuées sur des bases en dur, établies à l'aplomb des exploitations. Il s'agissait d'une double ligne parallèle de bornes bétonnées, implantêes en quinconce, et à des distances telles que les figures formées par trois bornes voisines étaient des triangles équilatéraux d'environ quinze mètres de Iongueur.

Très fréquemment, et souvent journellement, on effectuait un nivellement de précision du sommet des bornes (précision du mm autant que possible) et on mesurait avec la même précision les côtés de tous les triangles ci-dessus définis.

Après plusieurs campagnes de mesure, on a pu décrire de manière plus précise les lois des affaissements de surface au-dessus des mines de fer.

- L'affaissement total des terrains au-dessus d'une couche unique exploitée, ou au-dessus de deux couches exploitées simultanément est de 50 \% au maximum après stabilisation.

- Lorsqu'il s'agit de l'exploitation de deux couches superposées, l'affaissement relatif à la deuxième couche exploitée peut atteindre 65 \% de la puissance de cette couche.

- Les angles d'influence, c'est-à-dire les angles sur la verticale, des surfaces limites des zones perturbées, atteignent des valeurs comprises entre 15 et $25^{\circ}$ pour les exploitations en couche unique. $L^{\prime}$ application de ces angles permet de définir des zones où ne seront observés aucun affaissement ni aucune déformation horizontale.

En acceptant quelques déformations horizontales, qui peuvent être absorbées sans grands dégâts par des constructions de faible importance (maisons individuelles), on peut abaisser les angles à $10^{\circ}$ pour les couches peu profondes $(<135 \mathrm{~m})$ et $20^{\circ}$ pour les couches les plus profondes (environ $230 \mathrm{~m}$ ).

Les délais de tassement des terrains sont les suivants :
Si on appelle S le plein affaissement ci-dessus défini (par exemple $0,50 \mathrm{~h}$ ), on atteint :

$0,95 \mathrm{~s}$ en 66 semaines pour un recouvrement de $130 \mathrm{~m}$, en 77 semaines pour un recouvrement de $175 \mathrm{~m}$ en 96 semaines pour un recouvrement de $215 \mathrm{~m}$.

En extrapolant les lois des déformations en fonction du temps, on trouve que pour atteindre $0,98 \mathrm{~s}$, il faut :

2,5 ans pour un recouvrement de $130 \mathrm{~m}$

3 ans pour un recouvrement de $175 \mathrm{~m}$

4 ans pour un recouvrement de $215 \mathrm{~m}$.

Tous ces résultats permettent de définir les précautions à prendre pour la construction en terrains miniers.

En particulier, pour définir les limites de la zone de l'exploitation à traiter par une méthode spéciale (quí garde la stabilité), il faut, sur un plan de superposition fond-jour :

- tracer les limites au jour de la zone à protéger,

- dilater ces limites, tout autour de la zone, d'une

longueur

$\mathrm{d}=\mathrm{H} \operatorname{tg} \gamma$

- H étant la hauteur totale du recouvrement audessus de la couche exploitée,

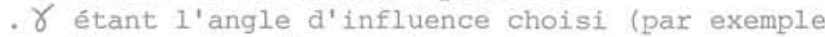
$20^{\circ}$ ) en fonction de la hauteur du recouvrement et de l'importance des immeubles â protéger.

I-1-4 - Méthode d'exploitation à l'intérieur des stots

Comme nous l'avons indiqué, il n'est pas question, en gếnéral, de procéder à une exploitation par dépilage complet dans les zones définies comme stots de protection. On pratique donc une méthode comportant des piliers abandonnés de manière à conserver la stabilité de la structure souterraine.

Les deux paramètres à définir sont, le taux de défruitement et la forme des piliers abandonnés. La détermination du taux, qui parait simple à priori, est en définitive un problème de géomécanique assez complexe.

En effet, l'idée simple, qui a été apoliquée traditionnellement par les ingénieurs est de considérer le recouvrement comme un ensemble pesant (dont il est facile d'évaluer le poids) et d'admettre qu'il applique aux piliers restants, une charge uniformément répartie. On en déduit donc la contrainte de compression sur les piliers,

$$
\rho_{\mathrm{gH}} \frac{1}{1-\mathrm{t}}
$$

$P=$ masse volumique du recouvrement

$\mathrm{H}=$ hauteur du recouvrement

$\mathrm{t}=$ taux de défruitement

qu'on peut comparer à la résistance à l'écrasement du minerai obtenue en laboratoire.

Malheureusement, ce raisonnement simpliste ne fait pas entrer en ligne de compte toute une série de considérations qui se sont dégagées progressivement avec le développement de la géomécanique :

- importance de l'effet d'échelle,

- importance de l'effet de forme des piliers,

- hétérogénéité et état de fissuration du minerai, 
- vieillissement des parois de piliers au voisinage desgaleries vides, et qui se traduit par un écaillage des parois et une diminution corrélative du noyau central résistant.

- enfin, et c'est le plus important : effets des surcharges imposées à la structure par le défruitement des zones voisines qui, par définition, sont intégralement dépilées (puisqu'on n'est plus dans le stot).

Depuis un certain nombre d'années, cet effet du voisinage des zones dépilées, a eté pris en compte dans l'établissement des stots, en laissant subsister à

la pêrıphérie, des bandes fermes très peu défruitées (maximum $20 \%$ ), et de largeur suffisante pour encais. ser à elles-seules, les surpressions des dépilages

Une autre manière d'exploiter les stots consiste à y pratiquer, dans des zones réduites du dépllage intégral, en laissant à la limite de ces zones, ou ilots, des culées, c'est-à-dire des bandes fermes, très peu défruitées, qui servent d'appui aux voûtes naturelles qui se constituent dans le recouvrement au-dessus des ilots.

I'éboulement des premiers bancs, qui se prođuit après le torpillage, constitue un remblai qui grâce au foisonnement, vient contrebuter les bandes fermes, et contribue largement à leur résistance dans le temps.

Cette méthode des ilots, qui était pratiquée depuis longtemps dans les stots à grande profondeur (audessus de $130 \mathrm{~m}$ ) fait l'objet actuellement d'une étude très approfondie, dont les premiers résultats seront présentés đans la conférence de M. TINCELIN.

\section{I-1-5-Problème de la stabilité des stots}

En conclusion des considérations ci-dessus, il apparaît que les stots des mines de fer, qui ont été réalisés au cours du temps, et en fonction des connaissances de l'époque de leur création, ne présentent pas tous les garanties de stabilité qu'on attendait. Il est même arrivé que certains d'entre eux soient le siège de mouvements de surface importants, qui ont détérioré des maisons et justifié une évacuation de certains quartiers menacés. Heureusement, jusqu'à présent, aucun incident de personne n'a été à đéplorer.

Dans ces conditions, il arrive qu'il soit nécessaire notamment pour les stots anciens d'assurer une surveillance des mouvements de terrains au jour et au fond, de manière à prévoir, dans la mesure du possible, les affaissements des terrains de surface.

\section{I-2 - Stabilité des chambres de dépilage}

La chambre de dépilage est la phase ultime de récupération du minerai

(voir figure 1)

et sa stabilité doit être garantie pendant une période brève.

Les règles d'exploitation ont été établies de manière empirique ; et, malgré leur validité et l'expérience du mineur, on a eu à déplorer plusieurs éboulements spontanés et imprévus du toit. Diverses parades ont été mises au point ; l'une d'elles consiste en un systême de contrôle et d'alarme permettant d'évacuer la chambre en temps utile.

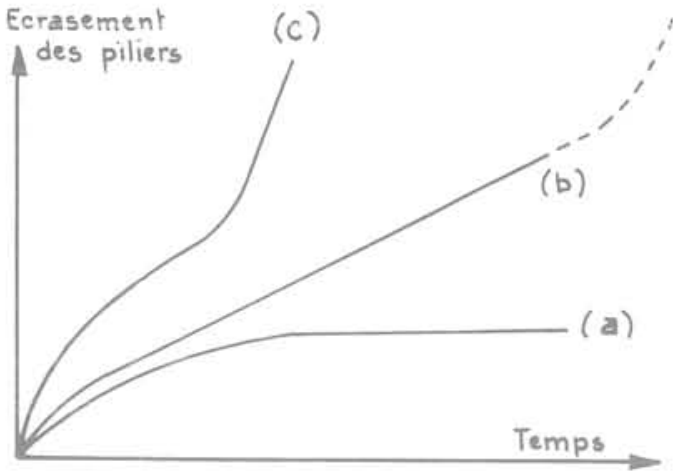

Fig.2 Courbe d'écrasement de piliers en compression mono-axiale

\section{I-3 - Comment évaluer la stabilité des piliers ?}

L'idée de départ a été de considérer que les piliers sont en compression mono-axiale, ce qui est une approximation grossière qui s'est révélée très fructueuse. Les courbes a'écrasement de ces piliers peuvent alors être assimilées à celles que l'on obtient dans lesessais en compression mono-axiale sur des échantillons cylindriques.

Trois allures de courbe sont possibles ( voir figure 2)

- courbe a : déformation décélérée ou a vitesse nulle : pilier stable.

- courbe b : déformation à vitesse constante positive non nulle : pilier en évolution, mais sans danger de destruction imminente.

- courbe c : déformation accélérée : pilier dont la destruction peut être imminente.

La vérification de ces courbes nécessite des mesures fréquentes, précises et de longue durée.

\section{CHAPITRE II - MESURES DE DEFORMATIONS DANS LES PILIERS}

L'étude de la déformation des terrains est une đes activitếs importantes des services techniques des mines de fer depuis 1948. En effet, depuis cette époque, la plupart des recherches en matière de mécanique des roches - et notamment les perfectionnements apportés aux méthodes d'exploitation - ont été basées sur la mesure des déformations des terrains

- soit mesure de convergence entre toit et mur des galeries,

- soit mesure d'expansion de la roche au toit des galeries.

C'est donc ces types de mesures qui ont été employés pour évaluer les mouvements de terrains au fond, susceptibles d'annoncer des affaissements au jour.

Les capteurs employés, et les moyens de collecte des informations ont suivi pas à pas les progrès de la technologie disponible. Il est apparu qu'il est nécéssaire de procéder à ces mesures avec une précision supérieure au $1 / 10$ e de $\mathrm{mm}$. En fait, les appareils développés et décrits ci-après satisfont à cette précision puisqu'ils permettent des mesures au $1 / 100$ e de $\mathrm{mm}$.

Nous allons examiner les différentes solutions apportées successivement à ce problème par SAMIFER, avec leurs avantages et leurs onconvénients. 
II-1 - Principe des mesures, emblacement des cap-

La structure à surveiller est toujours constituée par un certain nombre d'apnuis - piliers de forme quelconque et à la limite bandes fermes - sur lesquels repose le recouvrement.

Il nous a toujours semblé évident que la stabilité de l'ensemble de la structure est liée à la tenue des niliers. C'est donc la déformation de ces derniers qui a été mesurée dans tous les cas.

Le varamètre important est le tassement, c'est-àdire le raccourcissement des piliers sous l'effet de la surcharge qui $y$ est appliquée.

Pour évaluer ce tassement, l'idéal serait de placer un convergence-mètre sur une verticale au coeur du pilier. C'est évidemment impossible dans une exploitation en couche unique.

On se contente donc :

- soit de placer des convergence-mètres entre toit et mur d'une galerie voisine, mais en les implantant le plus près possible du parement du pilier en créant même le cas échéant une saignée verticale dans le pilier pour rapprocher 1'appareil du coeur de celui-ci,

- soit de procéder à des mesures de déformations horizontales dans le pilier même, à l'aide d'un extensomètre quelconque.

\section{II-1-1- Les mesures de convergence verticale}

Les mesures de convergence verticale contre les parements du pilier, sont celles qui accusent la plus grande déformation - elles ont également I'avantage d'une grande simplicité de la mise en place du convergence-mètre,

Elles ont par contre l'inconvénient de reproduire non seulement le tassement du pilier, mais également une part de la déformation du toit. En effet, ce dernier, qui est assimilable à une dalle semiencastrée se déforme en prenant sa flèche non seulement au droit du vide de la galerie, mais également dans la partie s'appuyant sur les zones voisines du parement du pilier. C'est du moins le cas dans les terrains des mines de fer, où les bancs du toit ont une rigidité assez voisine de celle du minerai.

Autrement dit, une forte déformation relevée pourrait annoncer une chute du toit au droit de la galerie, aussi bien que le tassement du pilier. Cet inconvénient est moins sensible quand les appareils sont placés dans des galeries déjà anciennes, où le toit a pu trouver sa stabilité.

(voir figure 3 )

II-1-2- Les mesures de déformation horizontale

Ces dernières sont les plus fiables. Elles demandent une précision encore plus grande que les mesures de convergence. En effet, la dilatation horizontale est en général beaucoup plus faible que le tassement vertical, surtout si on implante la base de mesure au coeur du pilier, dans la zone oil la roche est confinée et travaille en triple étreinte.

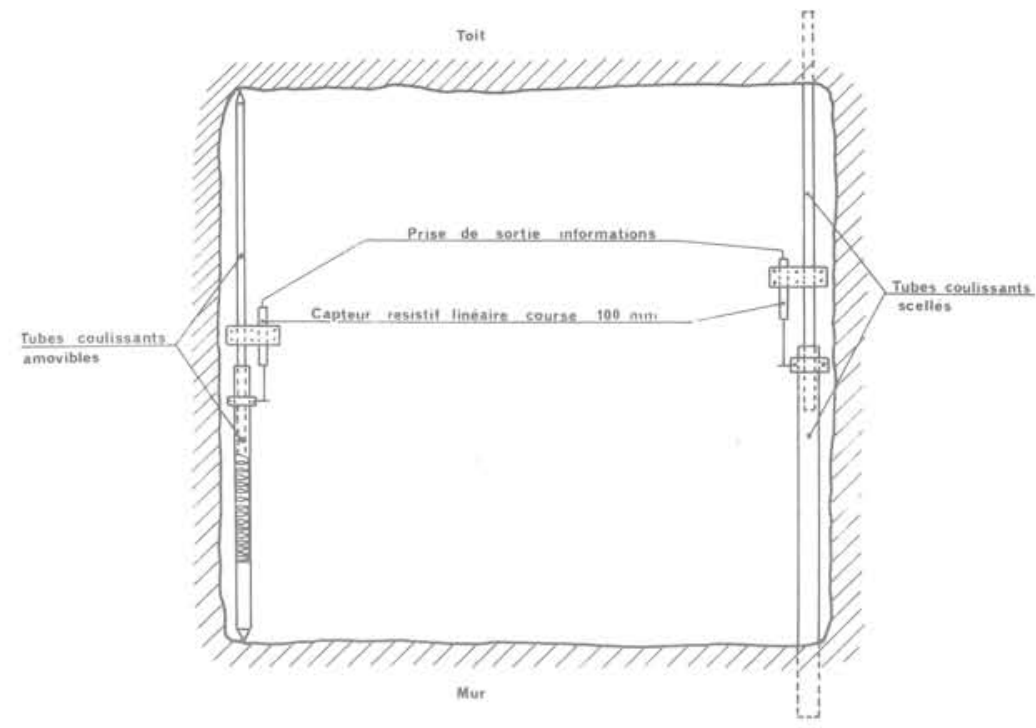

Fig.3 Convergencemètre électrique télescopique

De plus, à l'expérience, il est apparu, et les calculs aux éléments finis l'ont confirmé, que le sens de la déformation peut s'inverser suivant que la base est contenue complètement dans la zone centrale évoquée ci-dessus, ou si au contraire, ses extrémités sont placées l'une vers le coeur du pilier, l'autre au parement de la galerie voisine.

Dans le premier cas, on peut être amené à noter un raccourcissement de la base au fur et à mesure de la mise en compression du pilier. Cette déformation est alors un indice de stabilité. Lorsque la base centrale s'allonge, c'est au contraire un indice de déstabilisation.

$$
\text { (voir figure 4) }
$$

Pour les dilatomètres posés du coeur à la périphérie, on trouve toujours une dilatation qui tend à s'augmenter avec le tassement des piliers.

\section{II-2- Appareils de mesure utilisés et mis au point à SAMIFER}

II-2-1- Appareils de type mécanique

Ces appareils qui ont été mis au point il y a une vingtaine d'années par nos services, ont été utilisés pratiquement depuis lors pour toutes les mesures in situ effectuées dans le cadre de nos recherches de mécanique des roches. Ils ont déjà été décrits plusieurs fois, aussi serons nous brefs à leur sujet.

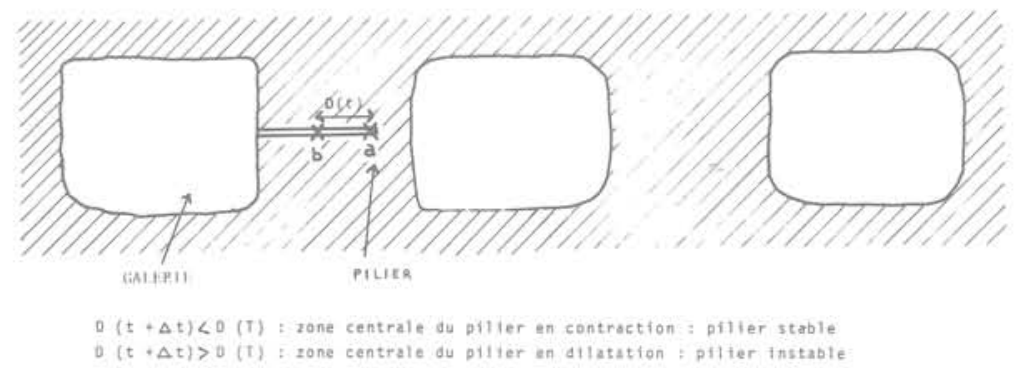

Fig. 4 Mesure de déformation horizontale. Etude de la variation de la distance a b. 


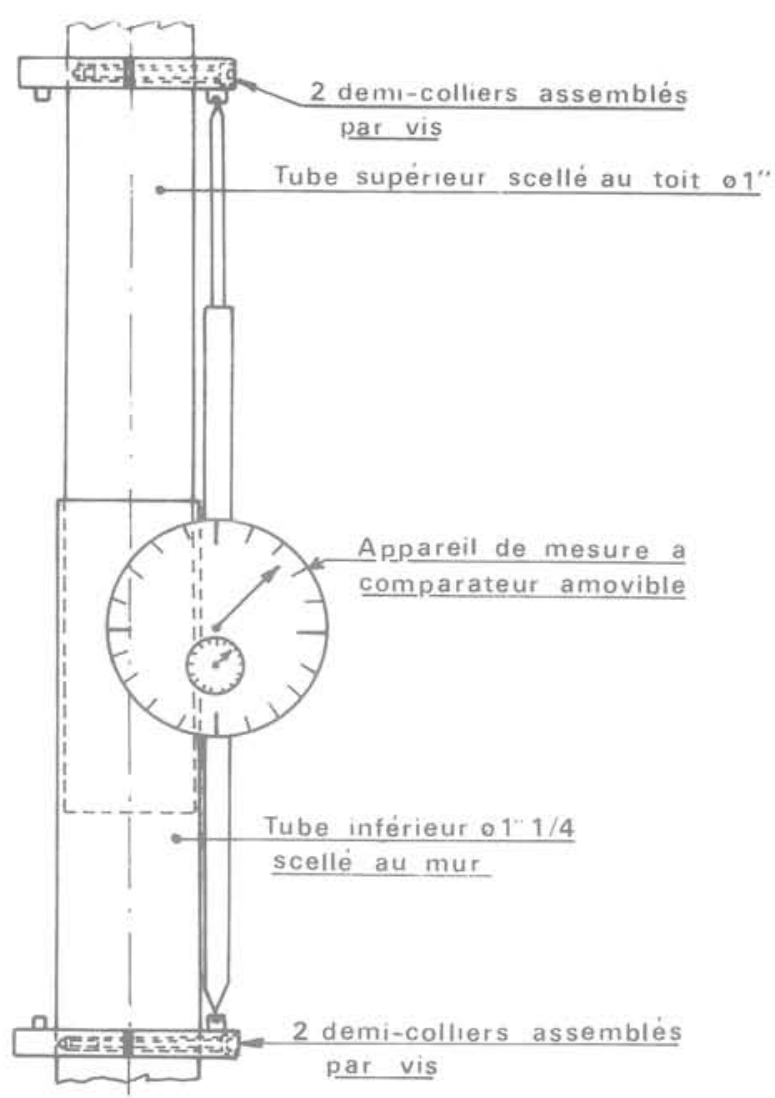

Fig. 5 Convergencemètre mécanique

La mesure repose toujours sur l'utilisation d'un comparateur mécanique à cadran qui n'est pas laissé sur place à la station de mesure, mais monté sur un bâti rigide amené à pied d'oeuvre à chaque visite par le technicien chargé des mesures.

La technique est légèrement différente suivant qu' il s'agit de mesures de convergence ou de mesures de dilatation.

\section{II-2-1-1- Mesure de convergence}

La station est constituée par deux tubes creux coulissant I'un dans I'autre avec le minimum de jeu. L'un de ces tubes est scellé dans le toit, l'autre dans le mur de la galerie. Tout tassement du pilier se traduit par une pénťtration plus grande d'un tube dans 1'autre. Cette pénétration est mesurée par le raprochement de deux plaques solidaires chacune de I'un des tubes. Les bases de mesures sont constituées par des plots très durs et protégés de la corrosion et des chocs, fixés sur ces plaques. Pour faire la mesure, on implante entre deux plots le support de comparateur équipé de ce dernier. La mesure se fait par comparaison avec une base étalon.

(voir figure 5)

II-2-1-2- Mesure de dilatation

On mesure la variation de la distance entre un point profond du massif et la face libre de la galerie voisine : une tige de longueur appropriée est scellée à la profondeur désirée dans le massif à observer. Une plaque de référence est solidarisée de la face libre du massif. L'extrémité libre de la tige est voisine de la plaque de référence. La distance

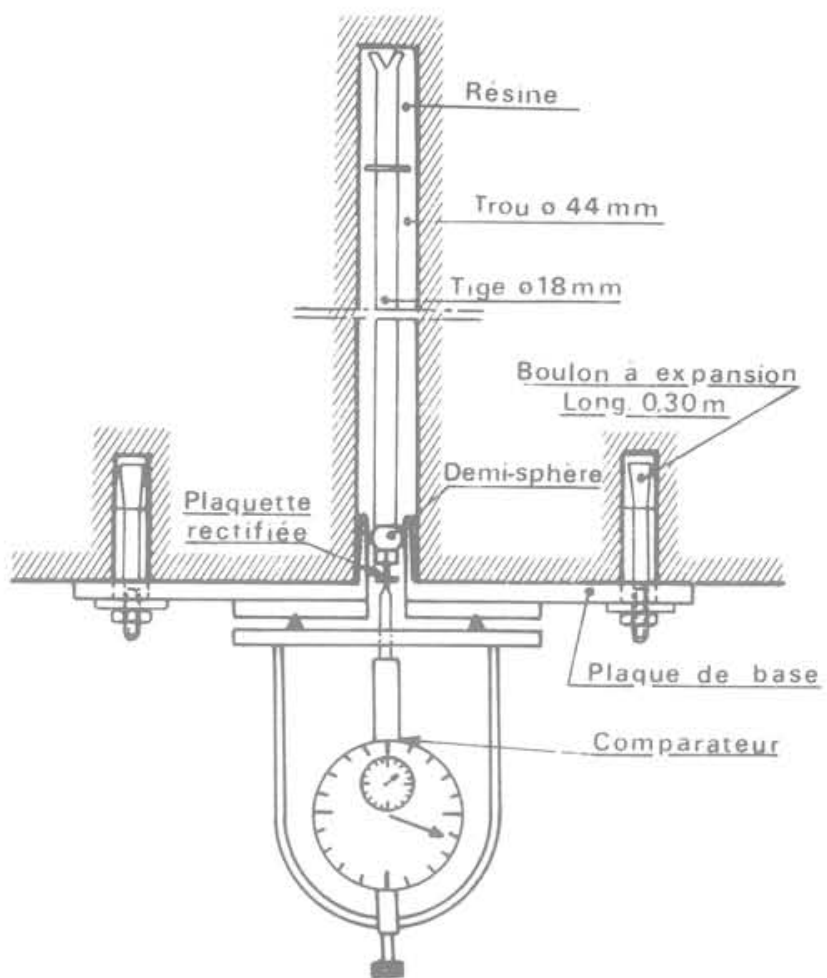

Fig.6 Dilatomètre mécanique

les séparant est mesurée à l'aide d'un comparateur de $30 \mathrm{~mm}$ de course et de $\pm 0,01 \mathrm{~mm}$ de précision. Le comparateur est solidaire d'un bâti-trépied permettant de faire trois mesures par rotation à $120^{\circ}$; la valeur obtenue en étant la moyenne. A chaque mesure, le comparateur est étalonné sur une base se trouvant dans le coffret de transport de l'ensemble. Notons qu'avec cet appareil il n'est pas possible d'implanter une base au coeur d'un pilier; pour accéder à cette mesure, il faut implanter tout près l'une de l'autre deux ou plusieurs tiges de différentes longueurs, et procéder par différenciation.

$$
\text { (voir figure 6) }
$$

\section{II-2-1-3- Inconvénients des mesures mécaniques}

Comme on l'a remarqué, ces mesures nécessitent pour chaque point de la courbe déformation/temns, une visite d'un technicien à la base de mesure. Il faut donc que celle-ci reste accessible sans danger, ce qui n'est pas toujours le cas.

Enfin, sur le plan de la surveillance, il n'est pas exclu à priori que des phênomènes importants puissent se passer entre deux visites, ce qui peut conduire à nerdre l'information essentielle susceptible d'annoncer une déstabilisation des terrains.

Il nous est arrivé effectivement d'avoir un éboulement d'un quartier exnloité par piliers abandonnés (hors stot, heureusement) survenu nendant un arrêt prolongé de la mine. Les mouvements des piliers qu' on avait notés avant l'arrêt étaient encore trop faibles nour qu'on puisse en déduire l'imminence de 1 'éboulement. 


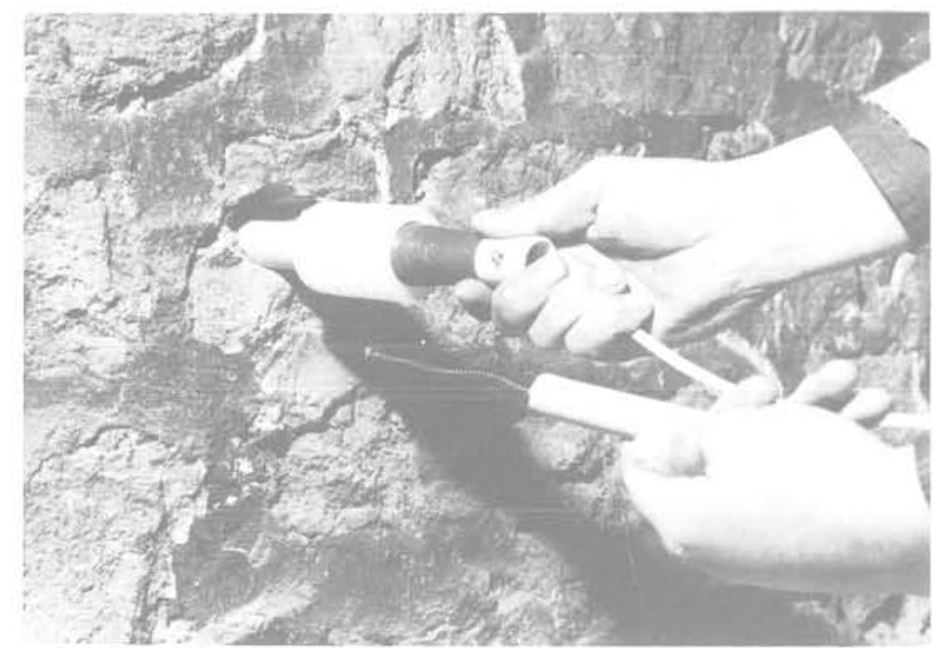

Fig. 7

II-2-2- Appareils de mesure électrique

II-2-2-1- Capteurs

Ces appareils ont été mis au point pour pallier si possible les inconvénients soulignés ci-dessus. La structure de base de la mesure - nour la convergence ou nour la dilatation - est toujours la même. On a simplement fixé sur l'ossature un capteur potentiométrique, dont les éléments sont resnectivement solidaires des parties de structure dont le mouvement relatif est proportionnel à la déformation.

(voir figure 7)

Le capteur est constitué par une résistance en forme de piste en plastique conducteur, sur laquelle circule un curseur, l'ensemble constituant un potentiomètre de résistance totale $10 \mathrm{k} \Omega$. La résolution de ce potentiomètre est "infinie", et sa résistance à l'humidité est suffisante pour qu'on puisse le laisser à poste fixe sur la base de mesure.

L'expérience a montré avec 5 ans de recul que les appareils en atmosphère saturée en humidité sont toujours fiables. A titre d'exemple, le nombre d'appareils posés depuis Avril 1976 a été de 90 et la cadence de pose actuelle est supérieure à 20 .

\section{II-2-2-2- Mesure}

La mesure de la grandeur recherchée est obtenue par une mesure de tension sur un circuit incluant le potentiomètre et alimenté par une source de tension connue parfaitement stable.

SAMIFER a mis au point un appareil, le Dicomètre SAM VD 78 , qui donne directement la longueur cherchée sous forme numérique en centièmes de millimètres (précision du capteur).

Le Dicomètre comporte à la fois le circuit d'alimentation du capteur, le circuit de mesure, et la conversion analogique digitale.

La forte résistance totale du capteur potentiométrique permet de le brancher sur un circuit de grande longueur permettant la mesure à distance.

II-2-2-3- Utilisation des capteurs

L'installation des capteurs potentiométriques peut être faite :

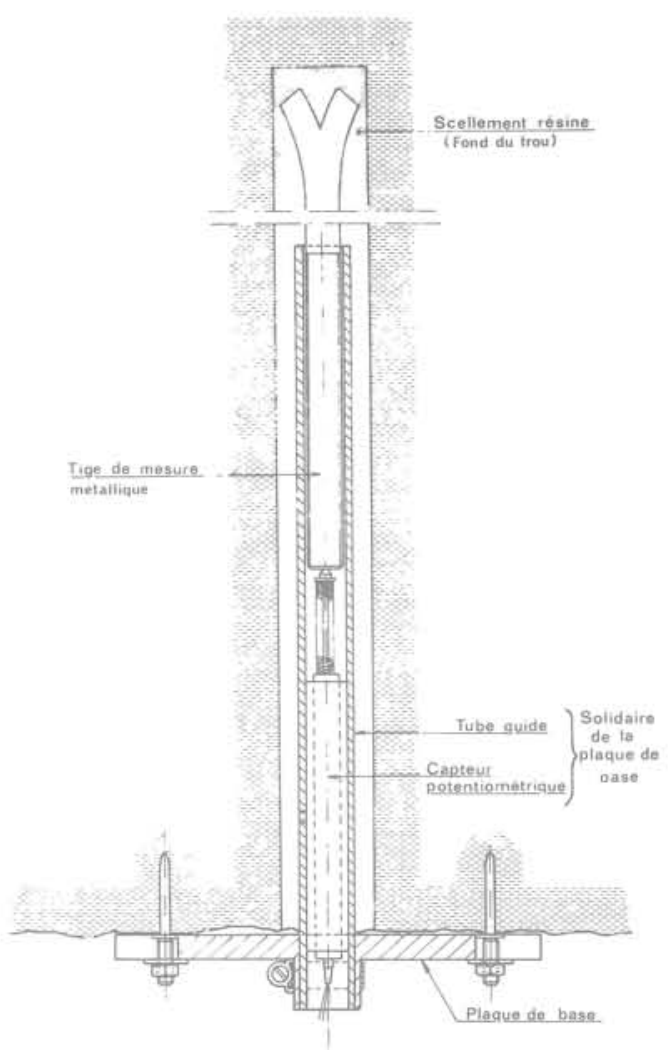

Fig.8 Dilatomètre électrique

à tige scellée et plaque

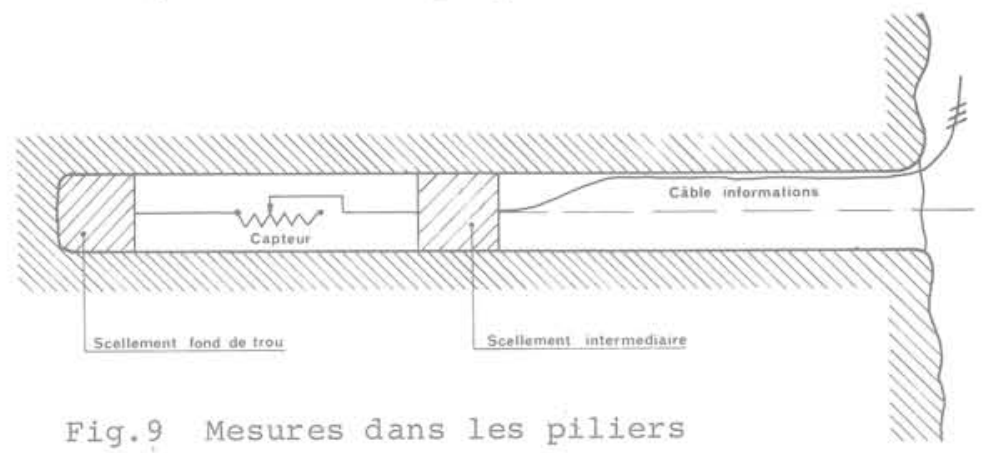

- soit sur des dilatomẽtres placés dans des trous de mine comme pour des mesures mécaniques, (voir figure 8)

- soit sur des convergence-mètres analogues à ceux des mesures mécaniques,

- soit sur des extensomètres à câble.

De plus, l'utilisation de capteurs potentiométriques a permis de résoudre un problème qui n'avait pas trouvé de solution avec les appareils mécaniques : la mesure d'une déformation au coeur du pilier.

C'est une simple adaptation de la tige du dilatomètre classique, permettant de 1'implanter entre deux scellements distincts placés dans un même forage, au coeur du pilier. La difficulté à résouare a été la réalisation des scellements, à une distance importante de $I^{\prime}$ orifice du trou. La liaison avec $I^{\prime}$ extérieur pour permettre les mesures est assurée simplement par un câble biconđucteur qui sort du trou. (voir fugure 9). 


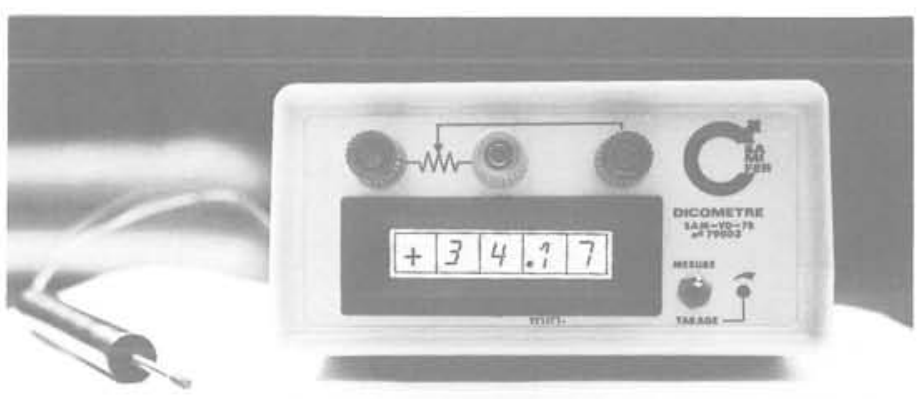

Fig.10 Dicomètre

\section{CHAPITRE III- UTILISATION ACTUELLE DES NOUVEAUX CAPTEURS POUR LA SURVEILIANCE DES STOTS}

Depuis la mise en oeuvre des capteurs potentiométriques, les mesures de surveillance des stots se font toujours à distance.

Cela permet :

- de rassembler I'arrivée des circuits en un Iieu unique, d'où gain de temps considérable. La seule précaution nécessaire est de bien renérer les prises de connexion de l'appareil de mesure pour bien identifier le capteur correspondant.

- d'éviter au technicien d'accéder aux bases de mesure, ce qui améliore beaucoup la sécurité de cette opération.

La difficulté principale des télémesures est la protection des câbles contre les chutes de blocs isolés ou les éboulements massifs. Quand un câble est coupé, l'information est évidemment perdue.

\section{III-1 - Dicomètre}

Si on ne désire que des mesures ponctuelles dans le temps, les seules qui étaient possibles avec les appareils méćaniques, on utilise le Dicomètre, dont l'emploi est beaucoup plus simple que le comparateur mécanique - et qui est d'ailleurs aussi plus rapide. Il suffit de connecter le Dicomètre sur les bornes des mesures d'un capteur, mettre l'appareil sous tension, et la longueur mesurée s'affiche immédiatement en valeur numérique : millimètres, dixièmes et centièmes. Une position "tarage" permet soit d'ajuster le zéro, soit d'avoir une mesure de référence pour corriger le résultat en fonction de I'état de I'anpareil.

$$
\text { (voir figure 10) }
$$

Jusqu'à plusieurs kilomètres de ligne, la longueur de celle-ci n'influe en rien sur la précision de la mesure.

\section{III-2 - Mesures en continu}

Le canteur électrique a apporté une autre amélioration, bien plus importante : la possibilité de suivre l'évolution des piliers en permanence si nécessaire. Deux techniques sont alors employées.

III-2-1- Enregistreur

On peut très simplement enregistrer en permanence la déformation relevée par le potentiomètre : pour

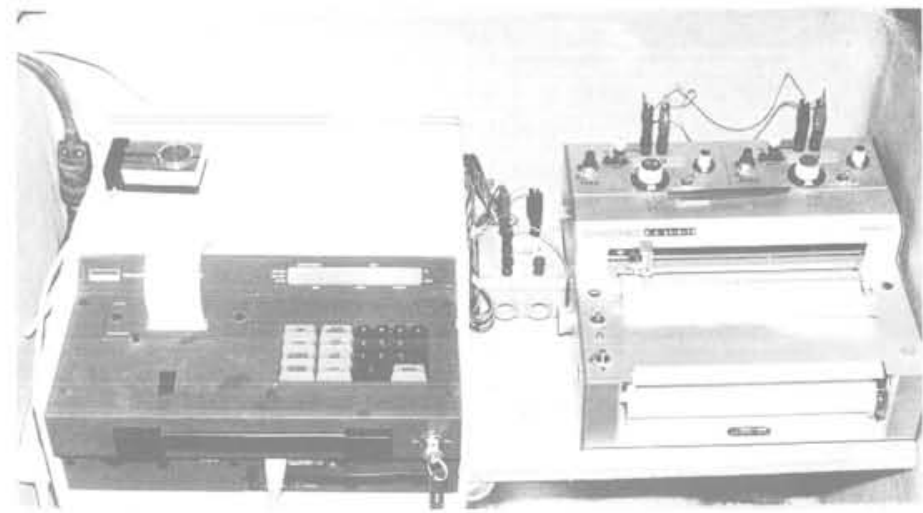

Fig.11 Centrale de mesures

ce faire, nous disposons d'enregistreurs de haute fidélité - en général à deux pistes - sur papier. Ces appareils sont dotés d'amplificateurs à très hautes performances, ce qui permet de dilater la déformation à volonté, en fonction du varamètre étudié. De même, la vitesse de déroulement đu papier est réglable. Ces appareils sont très sensibles, et ont une constante de temns relativement faible, ce qui vermet de noter même des transitoires de durée largement inférieure à sa seconde.

\section{(voir figure 11)}

C'est grâce à ces appareils que nous avons pu mettre au point les appareils d'alerte, type syaleb, dont nous parlerons ci-dessous.

\section{III-2-2- Centrale de mesures}

Les enregistreurs ne permettaient jusque récemment, que de relever les informations de deux capteurs (deux pistes). Une autre technique est employée lorsqu'on veut surveiller toute une batterie de capteurs, il s'agit d'un appareil que nous appelons "centrale de mesure". Il s'agit d'un petit ordinateur, qui explore, suivant un programme qu'on lui fixe à l'avance, tous les circuits qui lui sont connectés. Il dispose d'un convertisseur analogique -numérique, qui redonne directement en valeur digitale, l'information reçue. Celle-ci est transcrite immédiatement sur une bande de papier par une imprimante.

La durée minimale du relevé d'une mesure est de 15 s., l'appareil peut suivre jusque 20 circuits distincts. Il dispose d'une horloge interne, qui lui permet, si on le désire, de noter I'heure de chaque relevé.

L'échelonnement des relevés peut être fixé, dans le programme, entre $1 \mathrm{mn}$ et $24 \mathrm{~h}$.

Lorsque cette centrale a été employée, elle était installée au jour, dans un bureau, et était reliée aux capteurs par des câbles de plusieurs kilomètres. Les valeurs numériques notées par 1'imprimante sont relevées périodiquement et reportées sur des courbes.

C'est l'examen de ces courbes qui permet de vérifier si la structure surveillée est stable. 
CHAPITRE IV - RESULTATS OBTENUS DANS LA SURVEILLANCE DES STOTS ET DES EXPLOITATIONS PAR PILIERS ABANDONNES

Depuis 1967 de nombreux stots ou exploitations par piliers abandonnés dans des mines de fer, principalement, mais également dans des mines de bauxite de sel et de $\mathrm{Pb}-\mathrm{Zn}$, ont été ou sont encore actuellement surveillés en utilisant des appareils de mesure SAMIFER type convergence-mètre. En général, les déformations ont êté très faibles ou même nulles. Mais nous avons quelques cas d'éboulements dans des zones où une campagne de mesures était en cours.

Il s'agit, en général, de mesures mécaniques ; les mesures électriques ne sont utilisêes que depuis 1976.

Ainsi, nous avons pu étudier les trois cas de figure évoqués dans le chapitre I-3.

IV-1- Structures stables

De nombreuses campagnes de mesures ont été déclenchées à la demande des tuines à czuse de l'effondrement d'une partie de l'exploitation. Les zones surveillées se sont très souvent révélées stables, c'est-à-dire que les déformations mesurées étaient très faibles, de l'ordre de $1 / 100 e$ de mm sur plusieurs années, ou même nulles.

Dans le cas des stots, nous pouvons citer les mines de fer de Moutiers et Errouville, la mine de sel de Varangeville. Pour ce qui est des exploitations par piliers abandonnés, nous citerons les mines de fer de Droitaumont, Giraumont, ottange, Saizerais, et la mine de plomb-zinc de Largentière.

Ainsi, le stot de la mine de Moutiers concernait une zone exploitée en couche grise nar piliers abandonnés avec un taux de défruitement de $48 \%$.

(voir figure 12)

La campagne a duré d'Avril 1968 à Aolt 1980 ; et sur ces 12 ans, la déformation totale a été de $0,12 \mathrm{~mm}$, soit $1 / 100$ de $\mathrm{mm}$ var an.

Pour ce qui est de la fiabilité du matériel utilisé, il faut noter que la zone où étaient installés les convergence-mètres, a été inondée pendant 6 mois, et que 1'anpareillage a très bien résisté.

IV-2- Structures en évolution

Comme exemole de structures en évolution non dangereuse, c'est-à-dire que l'écrasement des piliers se fait à vitesse constante non nulle, on peut citer le cas d'un quartier de Saizerais où la campagne de mesure a débuté en Février 1968.

Il s'agit d'une exploitation par niliers abandonnés avec un taux de défruitement de 53 \% à 65 \%. Pendant 4 ans, les piliers n'ont cessé de s'écraser à vitesse constante; et il faut noter que cette zone n'est toujours pas effondrée.

$$
\text { (voir figure 13) }
$$

\section{IV-3- Structures instables}

Un certain nombre de campagnes de mesures ont permis de prévoir des éboulements. C'est le cas des mines de Rochonvillers, Bazailles (exploitation par élargissages), Moineville, Joudreville.

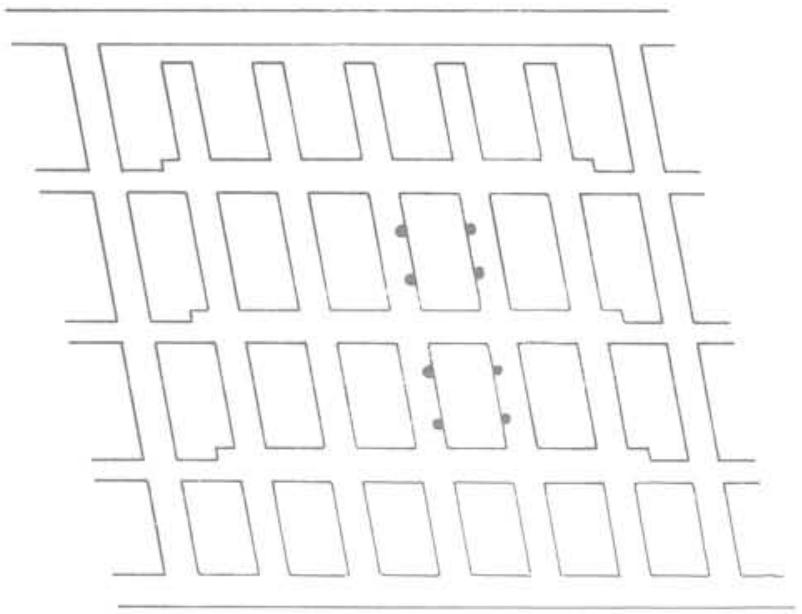

- Converqencemetres

Fig.12 Implantation des convergencemètres pour la surveillance du stot de Moutiers

Etudions plus narticulièrement le cas de la mine de Rochonvillers; Les mesures ont été effectuées dans un quartier exploité par chambres et piliers à $65 \%$.

$$
\text { (voir figure 14) }
$$

Sur les courbes, on note une nremière accélération le 4 Décembre 1973, suivie d'une accalmie. A nartir du 17 Décembre, la déformation s'accélère à nouveau. Les mesures sont arrêtées le 22 Décembre par crainte d'un éboulement qui se produit effectivement entre le 22 et le 27 Dếcembre 1973. Il aurait śvidemment été intéressant de continuer les mesures; mais il faut rappeler que ces mesures étaient faites à la main : on a donc da les arrêter pour ne pas risquer la vie d'un mineur.

Il faut retenir que l'alerte a été donnée une première fols le 4 Décembre et une deuxiême fois le 17 Décembre.

\section{CHAPITRE V - SYSTEME D'ALERTE}

A partir du moment où la technologie utilisée permettait d'enregistrer de manière continue les courbes de déformation en fonction du temps, il devenait non seulement possible, mais également souhaitable d'étudier avec soin les lois qu'on pouvait éventuellement en déduire, afin de parvenir à une "anticipation" permettant de prévoir de manière sûre une rupture.

Etant donné que les éboulements de stot sont des phénomènes très rares, et que l'exploitation est conduite de façon à les éviter, mais que, par contre, dans les chambres de đépilage, l'éboulement est recherché, cette dernière structure a été utilisée pour déterminer les paramètres pouvant constituer une alarme vis à vis des éboulements, et fixer leurs valeurs. 


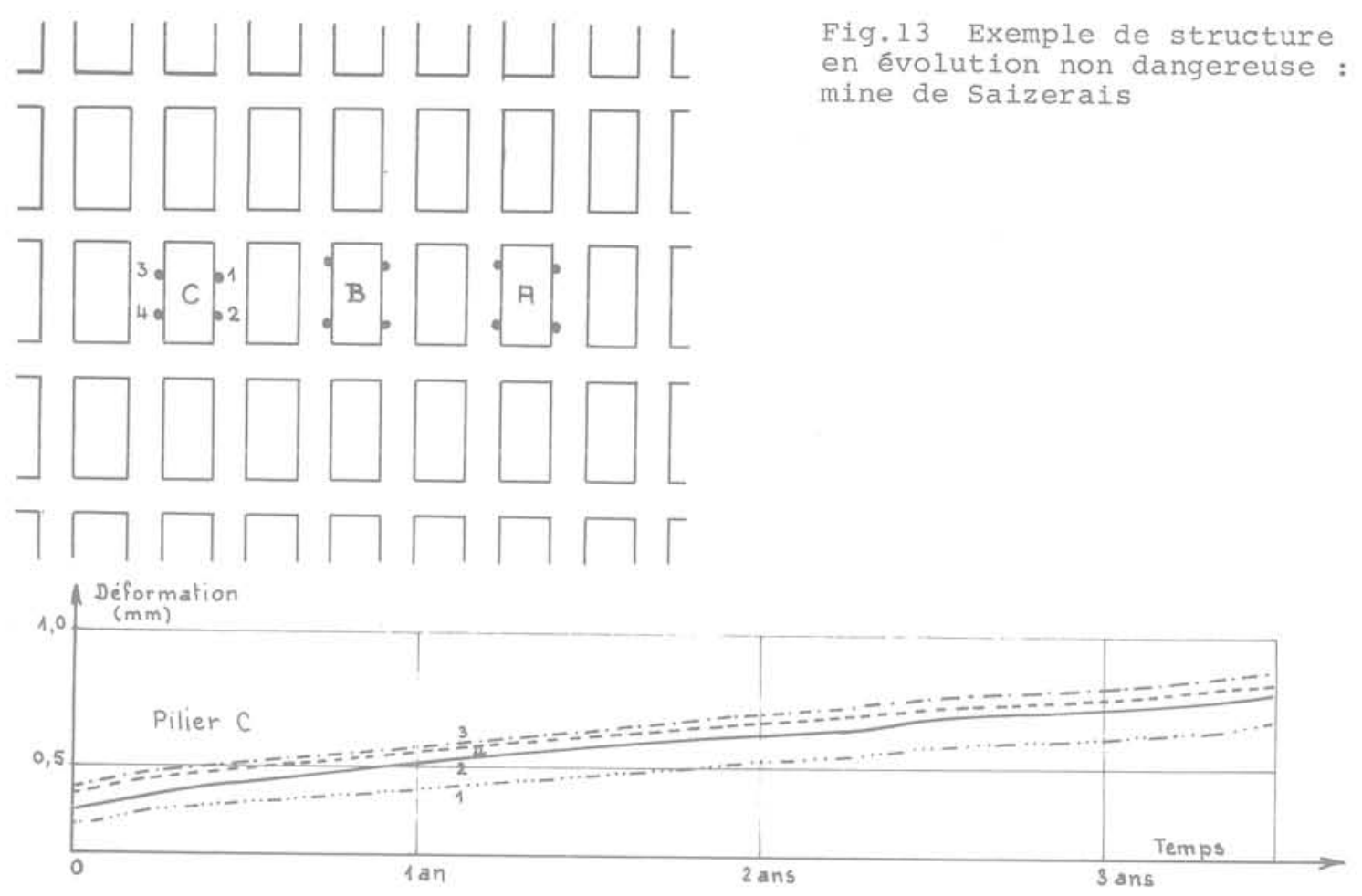

Fig.14 Exemple de structure instable : mines de Rochonvillers

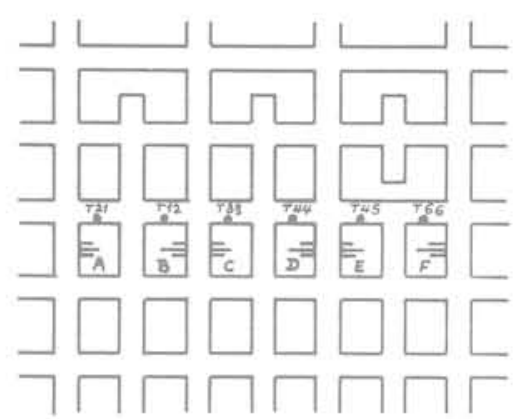

- Convergencemètres placés au ras des piliers

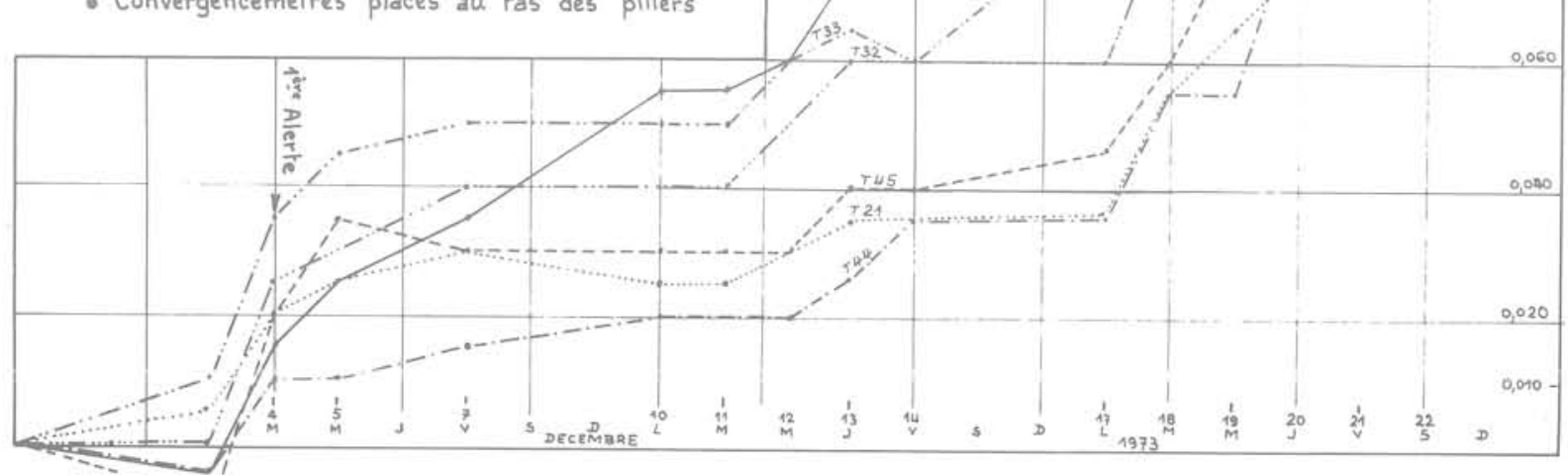




\section{V-1- Alerte dans les chambres de dépilage}

Cette recherche a été entreprise par SAMIFER depuis 1977. Pour des raisons d'urgence, et également parce qu'il rendait plus facile le contrôle et l'expérimentation, le premier champ de recherche fut celui des chambres de dépilage.

\section{(voir figure 1)}

Ces chambres, dont la vie est toujours brẽve, et se termine par le torpillage des appuis provoquant l'éboulement du toit, constituent des structures dont la stabilité reste encore aléatoire, malgré les progrès de la technique d'exploitation, et la grande expérience des mineurs.

Il s'agissait donc, à l'aide d'un système à imaginer, de prévoir un éboulement fortuit avec suffisamment d'avance pour permettre l'évacuation du personnel et du matériel avant que survienne cet éboulement.

\section{V-1-1- Mesures préalables}

Dans une première phase, on procéda à une série d'enregistrements continus dans des chambres de dépilage qu'on avait au préalable déstabilisées en amaigrissant le plus possible les quilles résiduelles, afin d'obtenir, si possible, leur éboulement spontané.

Ces mesures furent réalisées au cours de 7 campagnes dans différentes mines du bassin.

L'exploitation des courbes enregistrées au cours de ces campagnes a permis de mettre en évidence les points suivants :
- Si le paramètre le plus sensible, donc le plus adéquat pour annoncer à I'avance un éboulement est la convergence totale, c'est-à-dire la variation de la distance entre toit et mur, la mesure de dilatation, clest-à-dire de la déformation relative des strates proches du vide minier, par rapport à celles qui sont en profondeur, permet également de prévoir I'imminence de cet effondrement. Or, ce dernier Jaramètre a sur le précédent, le gros avantage de permettre de placer les capteurs dans les terrains, à l'abri des chocs inévitables des engins d'exploitation.

- Les valeurs de la convergence ou de la dilatation ne sont pas à prendre directement en considération pour prévoir un effondrement, en effet, les seuils d'alerte qu'il y aurait lieu de fixer sont très différents d'une mine à l'autre, et même d'une recoupe â l'autre. La vitesse de variation de ces paramètres en fonction du temps ne peut pas non olus être comparée à une valeur de référence sûre.

- Le meilleur paramètre à utiliser comme critère de stabilité est la dérivée seconde de la dilatation, en effet cette dérivée est nulle pour une zone stable; elle est négative (décélération) pour une zone qui se stabilise après un tir ou un éboulement placé à proximité ; elle est nositive (accélération) pour une zone déstabilisée dont l'éboulement est imminent. La difficulté est de mesurer ou de calculer ce paramètre, car ses valeurs critiques sont très faibles : de l'ordre de quelques dixièmes de microns/minute/minute soit $10^{-12} \mathrm{~g}$.

A titre d'illustration, nous donnons,

(voir figure 15)

Fig. 15

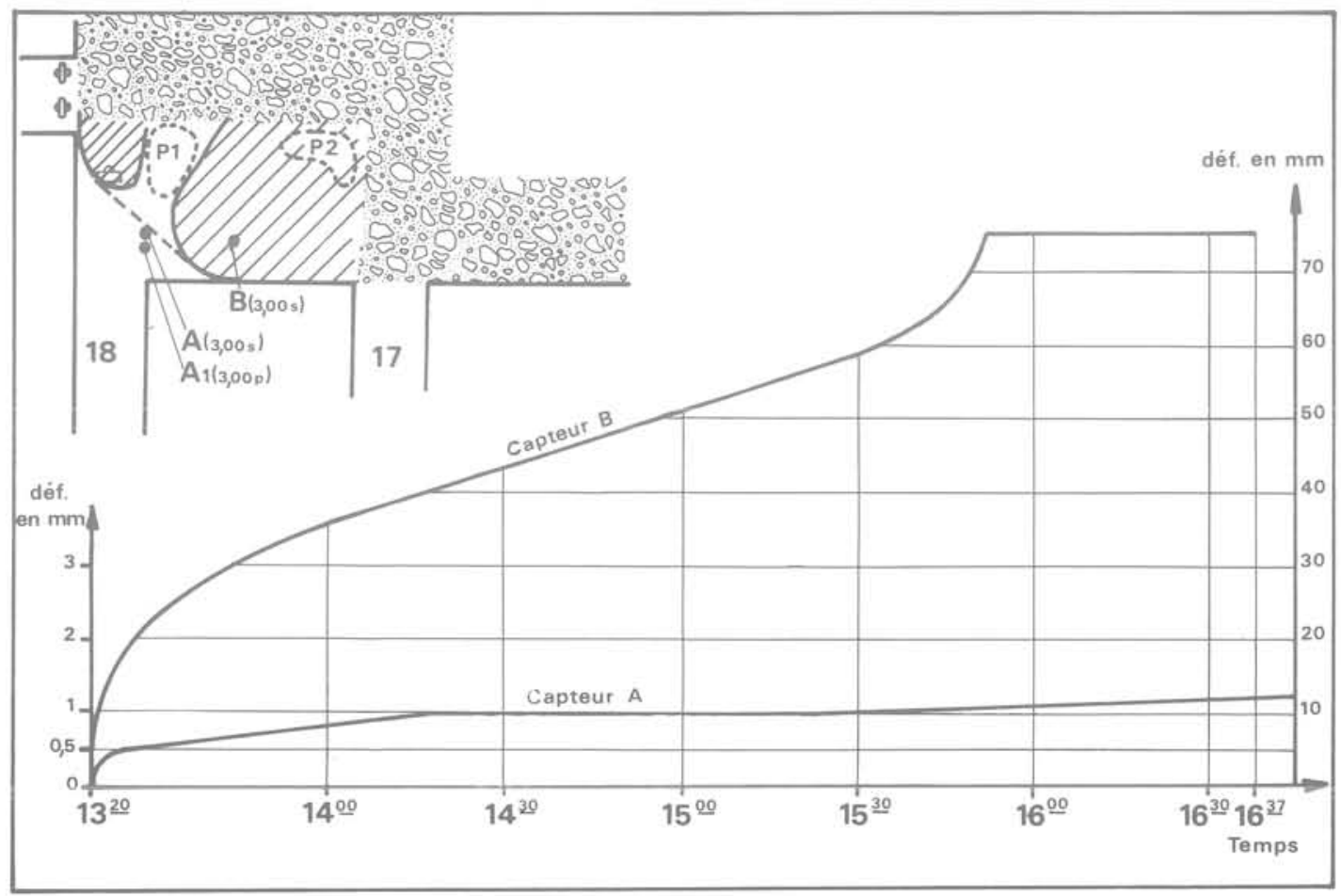


une courbe relevée au cours d'une des campagnes susmentionnées. Elle a été obtenue dans un dépilage en couche siliceuse noire, avec un toit très déformable. On avait installé deux dilatomètres, aux points $A$ et $B$ situés sur le plan en haut de la figure. Le graphique montre I'évolution de la dilatation du toit en fonction du temps. Le dernier tir dans la recoupe a eu lieu au temps $13 \mathrm{~h} 30$, et l'éboulement à l'instant entre $15 \mathrm{~h} 30$ et $16 \mathrm{~h}$ o0 oũ la courbe du capteur B est interrompue.

Ce graphique met en évidence les points suivants :

- La dilatation mesurée par le capteur B a augmenté très vite dans les instants qui ont suivi le tir. Elle s'est rapidement amortie, et, au bout d'une demi-heure sa vitesse d'accroissement s'est stabilisée à une valeur non nulle; celle-ci s'est accélérée â partir de $15 \mathrm{~h} 30$, pour tendre vers l'infini (en fait vers $g=$ accélération de la pesanteur) dans les instants qui ont précédé immédiatement 1 'éboulement.

- le capteur A qui n'était pas dans la zone intéressée par l'éboulement, a relevé une dilatation constante à partir de $14 \mathrm{~h} 15$.

Cette courbe, qui est semblable ã la plupart des enregistrements relevés au cours des campagnes de mesures, met bien en évidence les phénomènes de décélération lorsqu'après un tir, le toit évolue vers une certaine stabilité, et l'accélération qui précède l'éboulement.

La conclusion des campagnes préalables (effectuées en 77 et début 78) fut la décision d'utiliser l'accélération de la déformation comme critère annonciateur de l'éboulement.

\section{V-1-2- Etude du prototype d'appareil d'alerte}

\section{V-1-2-1- Analyse des enregistrements}

Après les 7 campagnes de mesures, SAMIFER était en possession d'un certain nombre de courbes analogues à celle que nous avons présentée. Toutes ces courbes montraient la possibilité d'utiliser l'accélération de la déformation comme critère d'alerte avant l'éboulement, mais elles montraient aussi qu'un simple calcul de l'accélération instantanée était inutilisable. Il fallait đonc, dès le départ, éliminer la simple dérivation électronique du signal délivré par le capteur.

Au contraire, il était nécessaire d'introduire la valeur mesurée comme donnée dans un processus de calcul de type informatique, susceptible, par des tests appropriés, de choisir le moment de donner l'alerte.

L'ếvolution récente des microcalculateurs nous donnait beaucoup d'espoir de succès, mais il était tout de même indispensable de mettre au point 1'algorithme qui servirait à traiter l'information.

Ce travail fut réalisé en partie par les ingénieurs de SAMIFER et complété par les spécialistes de la Société qui a assuré la construction du prototype. Le microprocesseur ne pouvait pas traiter le signal sous forme analogique, il fallait donc, dans un premier stade, le mettre sous forme digitale. Ce travail a été réalisé manuellement sur un certain nombre de courbes relevées au cours des campagnes.
Finalement, on a pu mettre au point un programme de calcul qui présente les caractéristiques suivantes :

- Il tient compte des données reçues depuis sa mise en service.

- Ces données ont, dans le calcul, un poids d'autant plus faible, qu'elles sont plus anciennes.

- Comme 1'appareil doit être opérationnel dès sa mise en oeuvre, il se recrée lui-même un passé virtuel à partir des toutes premières mesures relevées ; le calcul de l'accélération ne démarrant que $5 \mathrm{mn}$ après la mise sous tension du calculateur.

Ces caractéristiques sont toutes indispensables. Ainsi, comme on l'a vu, un tir provoque à coup sûr une grosse perturbation dans les déformations, qui se traduit, en général, par une forte accélération, suivie ã brève échéance par une décélération ; la mise en mémoire de ces données aberrantes entraine des perturbations inacceptables, qui ont pour effet "d'aveugler" 1'appareil. Il faut donc, au moment du tir, arrêter le calcul et le remettre en oeuvre après les grosses perturbations. La constitution et l'utilisation du "passé virtuel" permettent au Syaleb d'être opérationnel et fiable quelques minutes seulement après sa remise en service.

Sur le plan des principes, signalons encore qu'il a été décidé de comparer le résultat du calcul d'accélération à deux valeurs seuils :

- un seuil d'alerte qui indique à l'ouvrier que I'éboulement, sans être imminent, doit être envisagé comme probable; le dépaszement de ce seuil doit donc inciter tout le personnel à redoubler de vigilance,

- un seuil d'alarme, qui annonce l'imminence de l'éboulement, et signifie donc qu'il est urgent d'évacuer la chambre concernée.

\section{V-1-2-2- Simulation sur ordinateur}

Le programme a été testé sur ordinateur en utilisant les courbes de dilatation obtenues lors de la campagne de mesures d'expansion au fond décrite précédemment.

Ainsi, une vingtaine de courbes ont été dépouillées minute par minute (alors que le calculateur définitif sonde le capteur 64 fois par seconde, effectue la moyenne de ces mesures toutes les secondes, et utilise ces moyennes comme données pour le calcul de 1 'accélération avec un programme analogue à ceIui quí a été testé).

Cette simulation a permis de vérifier que le programme donnait des valeurs d'accélération satisfaisantes. De olus, on a pu sélectionner 4 valeurs de l'accélération au-delà desquelles la chambre évolue de façon irréversible, l'éboulement se produisant dans la demi-heure qui suit : dans chaque mine, on choisira en fonction de la nature du terrain un de ces seuils pour déclencher 1'alarme. Chacun d'eux étant doublé par un seuil d'alerte comme indiqué au paragraphe précédent.

\section{V-1-2-3- Caractéristiques des prototypes}

La recherche préalable a abouti à la construction 


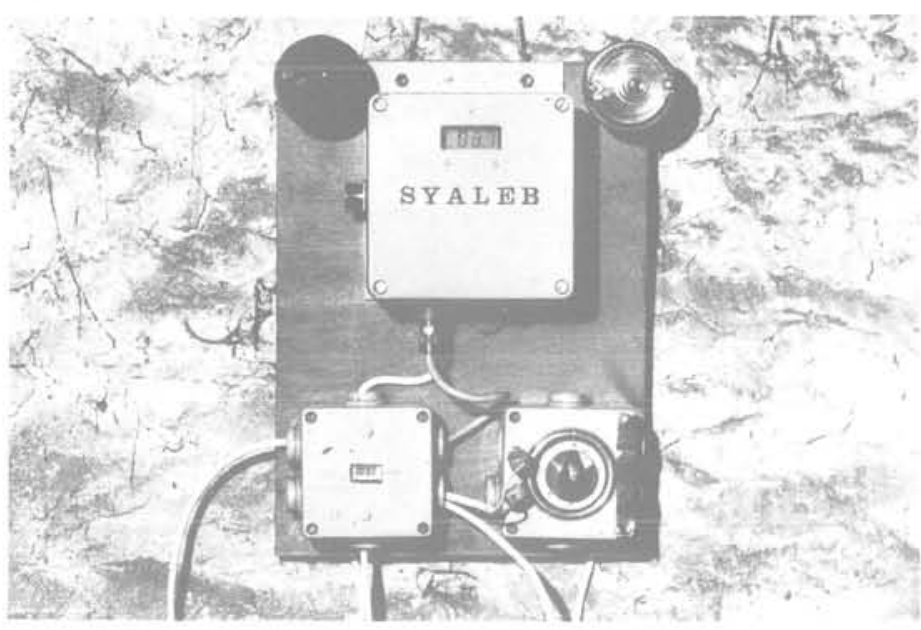

Fig. 16

d'une série de cinq prototypes d'un appareil qui a reçu le nom de "Syaleb", c'est-à-dire système d'alerte vis-à-vis des éboulements, et qui présente les caractéristiques suivantes : (le syaleb et notamment son procédé de traitement de 1'information, a fait 1'objet d'une demande de brevet, qui en est actuellement au stade documentaire) :

- il se présente sous forme d'un coffret aluminium étanche (160 mm X $160 \mathrm{~mm} \times 90 \mathrm{~mm}$ ), muni d'une plaque de fixation sur un tableau (voir photo).

(voir figure 16)

- il est alimenté par une batterie extérieure de 12 volts (deux connecteurs sont prévus pour permettre de changer la batterie sans interrompre le calcul).

- il est connectable à un seul capteur, qu'il alimente directement en tension stabilisée.

- il est canable de délivrer deux signaux distincts : un signal d'alerte, et un signal d'alarme, en fonction du résultat du calcul.

- il affiche en permanence, par dispositif à cristaux liquides, la valeur de la déformation mesurée par le capteur. Cet affichage permet également, par code approprié, c'avertir l'utilisateur des incidents tels que :

$$
\begin{aligned}
& \text { - tension de batterie trop faible, } \\
& \text { - tension d'alimentation du capteur insuf- } \\
& \text { fisante, } \\
& \text { - alerte donnée, } \\
& \text { - alerte donnée, mais tension de batterie } \\
& \text { faible, } \\
& \text { - alarme donnée. }
\end{aligned}
$$

- pour des prototypes uniquement, il comporte deux sorties permettant de les connecter à un enregistreur en vue de contrôler la valeur analogique de la déformation donnée par le capteur, et l'accélération calculée par le Syaleb.

L'appareil comporte essentiellement un microcalculateur qui a pour fonctions :
- de piloter la conversion numérique de l'information analogique fournie par le capteur, par l'intermédiaire d'un convertisseur digital analogique,

- de traiter l'information de déplacement (filtrage, calcul d'accélération, comparaison avec valeur de seuil),

- de fournir les ordres pour deux commandes d'alerte et d'alarme (fermeture d'un contact lorsque le seuil est dêpassé),

- de gérer l'affichage du déplacement,

- de vérifier la tension d'alimentation du potentiomètre.

Ce microcalculateur est monté de manière qu'on puisse modifier les seuils d'alerte - le seuil d'alarme est systématiquement fixé à la valeur double du seuil d'alerte.

On peut faire varier le seuil d'alerte entre 0,1 micron/minute et 0,8 micron, en quatre paliers, par doublement successif. En comparant ces seuils d'accélération à $\mathrm{g}$, on voit qu'ils peuvent évoluer entre 0,28 et $2,26 \times 10^{-11} \mathrm{~g}$. Ces chiffres permettent de se faire une idée de la sensibilité de l'appareil.

\section{V-1-2-4- Montage pratique}

En vue de son utilisation au fond, on a montë le coffret sur un tableau sur lequel sont fixés également (voir photo)

- le dispositif donnant l'alerte : pour l'instant, il s'agit d'un flash clignotant semblable à ceux qu'on voit maintenant dans certaines signalisations routières. Ces dispositifs délivrent des émissions lumineuses très intenses, mais de très courte durée, en sorte que l'éblouissement est pratiquement nul, et la consommation d'énergie faible.

- le dispositif d'alarme : une sirène modulée, qui peut être facilement entendue, malgré le bruit de fond relativement élevé qui règne dans les zones d'exploitation des mines de fer (on peut également utiliser un flash du même type que celui utilisé pour 1'alerte mais de couleur différente).

- un contacteur temporisé, qui permet de couper 1'alimentation du calculateur au moment des tirs, mais rétablit automatiquement celle-ci après un délai fixé à l'avance.

En plus du tableau, la station comporte évidemment une batterie d'alimentation de 12 volts. Pour 1'instant, on s'est contenté d'une batterie classique au plomb, type camion. Il ne nous semble pas nécessaire jusqu'à preuve du contraire, de prévoir un équipement plus élaboré, donc plus cher.

Il est bien entendu que les accessoires du Syaleb que nous venons de décrire pourront être très facilement modifiés si la nécessité s'en fait sentir au cours des essais. 


\section{V-1-3- Essais des prototypes}

Dès qu'ils furent réalisés, les prototypes furent soumis à une série d'essais :

- D'abord à des essais de laboratoire qui permirent de tester la validité du calcul en simulant, sur un banc d'essai mis au point spécialement pour ce travail, des déformations fictives dont l'évolution dans le temps comportait soit des lois simles (creneaux, sinusoĩdes, suites intempestives d'accélérations et de đécélérations), soit des lois semblables à celles qui étaient observables sur les enregistrements.

- Ce furent ensuite des essais en vraie grandeur, dans des chambres de dépilage, que l'on avait volontairement déstabilisées de la même manière que dans la phase des enregistrements préalables.

Les essais de laboratoire ont montré que le lissage effectué par le calculateur est très efficace - ils ont conduit à la décision d'arrêter le syaleb pendant les tirs, afin que l'accélération due à ceuxci ne masque pas une accélération ultérieure, qui serait, elle, annonciatrice d'un éboulement.

V-1-4- Résultats et conclusions

Les essais in situ ont confirmé la validité du Syaleb pour assurer la fonction d'alerte qu'on lui avait fixée. A 1 'heure actuelle, après traitement de 24 chambres de dépilage dans lesquelles on a pu obtenir 11 éboulements spontanês, qui peuvent seuls servir de référence, le syaleb a donné 1'alerte et 1'alarme dans tous les cas, l'alarme précédant l'éboulement d'un temps compris entre 3 et $60 \mathrm{mn}, 1 \mathrm{a}$ movenne se situant à $16 \mathrm{mn}$ ( 2 valeurs à $3 \mathrm{mn}$ et une à $4 \mathrm{mn}$ ) .

Nous considérons donc cet appareil valable, dans le domaine de la surveillance des chambres de dépilage, à condition qu'il ne soit pas considéré, du moins pour le moment, comme seul juge de la stabilité de la chambre. Nous estimons en effet qu'il ne dispense pas les mineurs et agents de maftrise de leurs observations habituelles concernant l'état du plafond et des appuis de la galerie en cause.

V-2- Utilisation du Syaleb à d'autres fins

Tel qu'il est réalisé maintenant, le syaleb est dono pratiquement opérationnel pour être utilisé dans les chambres de dépilage. Il commence d'ailleurs à être installé en veille, sans qu'il s'agisse spécialement d'essais, sauf à vérifier sa tenue dans 1'ambiance des mines. Dès que les premiers essais ont montré que le paramètre "accélération" pouvait valablement être comparé à un seuil fixe pour annoncer l'approche a'un éboulement, et que 1'algorithme de calcul du microprocesseur était opérant, on pensa évidemment à étendre 1'usage du syaleb à la solution d'autres problèmes.

\section{V-2-1- Essais en mini-taille}

Dans le cadre de la recherche sur la méthode d'exploitation par llots réduits (cf. conférence $n^{\circ}$ ), on se trouva en présence d'un site, en couche mince, à faible profondeur, où on constatait un retard dans l'éboulement du toit de l'arrière taille. Pour éviter d'être surpris par un éboulement intempestif qui aurait pu s'avérer dangereux, on avait placé au milieu de la taille un convergence-mètre à capteur potentiométrique relié à un Syaleb, avec enregistrement de la courbe. Le syaleb a donné des alertes chaque fois que I'accélération dépassait le seuil prévu et effectivement chaque alerte a été suivie d'un éboulement partiel. Le syaleb a donc bien rempli le rôle qui lui était dévolu.

\section{V-2-2- Installation pour stots}

Pour permettre la surveillance de plusieurs points en même temps, SAMIFER a conçu et fait réaliser un appareil capable de sonder par multiplexage jusqu'à dix circuits équipés chacun d'un potentiomètre. Les valeurs d'accélération sont calculées à chaque sondage d'un circuit. Pour la définition des valeurs d'accélération prises comme référence, on peut utiliser jusqu'à deux valeurs de référence distincte, chacune de ces valeurs étant affectée à une série de capteurs.

Si une valeur d'accélération supérieure à un des seuils est constatêe, l'alerte est donnée, et un voyant s'allume pour permettre de répérer sur quel circuit le dépassement de l'accélération a été constaté par le calculateur. Ceci permettra de juger de 1 'imminence et de l'importance d'un éboulement partiel ou total du stot et d'en déduire les risques d'affaissement en surface. 
